\title{
CRITERIOS DE LOS JÓVENES DEL DEPARTAMENTO DE CALDAS (COLOMBIA) ACERCA DE LA PARTICIPACIÓN EN POLÍTICA ${ }^{1}$
}

Páginas 63 - 86

\author{
CRITERIA OF YOUNG PEOPLE ABOUT THE POLITICAL \\ PARTICIPATION IN CALDAS STATE ${ }^{2}$
}

\section{José Rubén Castillo García ${ }^{3}$}

Frases claves: participación de los jóvenes en política; interés de los jóvenes por la política; pensamiento de los jóvenes sobre los partidos y los movimientos políticos; participación de los jóvenes en las elecciones; conocimiento de los jóvenes sobre las normas relacionadas con ellos.

Keywords: political participation of youth, interest in politics, viewpoints about political parties and movements, participation in elections, knowledge about laws related to them.

\section{Resumen}

Este documento es el resultado de una parte del estudio que se realizó con jóvenes en el Departamento de Caldas (Colombia). La investigación general, buscaba caracterizar la realidad juvenil en esta región del país con base en las áreas que definió la gobernación del Departamento de Caldas en el Decreto No. 0654 en 2007, mediante el cual adoptó la política pública de juventud. Estas áreas son: trabajo, salud, comunicación, educación, economía, deporte, recreación, cultura, familia, medio ambiente, la participación y política. Esta última área, es la que convoca las siguientes reflexiones.

Según el Departamento Administrativo Nacional de Estadísticas de Colombia (DANE, 2005) la población de jóvenes, estimada a 2007 para este departamento era de 237.130, (97.789 de 14 a 18 años, 74.413 de 19 a 22 años y 64.928 de 23 a 26 años). Por ello, para efectos del presente estudio, la muestra probabilística que se definió fue de 4.479, y las personas consultadas 4.559, discriminadas porcentualmente de los 27 municipios del departamento y ubicadas proporcionalmente con base en la población de cada municipio. Esto nos per-
1 Traducción de Carlos A. Muñoz T. Revisión Centro de Traducción del Instituto de Idiomas UAM.

2 Este artículo se apoya en los resultados de la investigación sobre la Realidad Juvenil en Caldas, desarrollada en el marco de las actividades del Observatorio de Juventud de Caldas, iniciada en enero de 2008 y terminada en diciembre de 2009 .

3 Docente investigador, Profesor Titular de la Universidad Autónoma de Manizales, Trabajador Social, Magíster en Desarrollo Educativo y Social y Doctor en Ciencias Sociales, Niñez y Juventud. Miembro de los grupos de investigación: "Ética y Política" de la Universidad Autónoma de Manizales; "Jóvenes, Culturas y Poderes" del Centro de estudios avanzados en niñez y juventud alianza CINDE-Universidad de Manizales y "Juventud y Nuevas Prácticas Políticas en América Latina" del Consejo Latinoamericano de Ciencias Sociales - CLACSO -. Dirección electrónica: jorca@autonoma. edu.co y jorca53@gmail.com

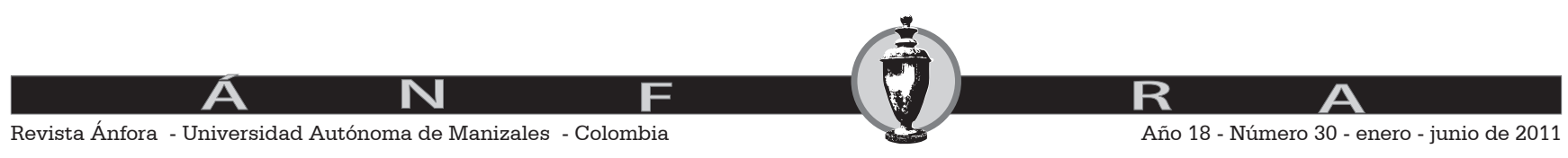


mite afirmar que las conclusiones son válidas para todos estos jóvenes, es decir, las personas comprendidas entre los 14 y los 26 años de edad, tomando como base lo indicado por la Ley 375 de 1997 o Ley de la Juventud en Colombia.

Los resultados se obtienen a partir de la base de datos que deriva de aplicar el programa de estadísticas SPSS (Statistical Package for the Social Sciences).

Con base en los datos producidos, se identifica y caracteriza lo que piensan estos jóvenes, sus intereses, motivaciones, puntos de vista en relación con la política y la percepción del país, de los partidos y movimientos políticos, el nivel de información que tienen acerca de las normas e instituciones relacionadas con ellos, y de las oportunidades que ellas les brindan.

El desarrollo de cada subtema, se distribuye con base en: 1) edad y género, 2) subregiones en que se divide el Departamento de Caldas: Centro sur, Norte, occidente alto, occidente bajo, Magdalena caldense y alto oriente; y 3) actividad que realizan: estudia, trabaja, estudia y trabaja, y, no estudia ni trabaja; y por la ubicación geográfica, referida a si están viviendo en la zona urbana o en la rural.

Esta información permite elaborar una primera descripción, y desde allí se hacen algunas aproximaciones conceptuales a cada uno de los temas sobre los cuales fueron consultados. Al final se presentan las conclusiones generales que se derivan de los planteamientos presentados por los jóvenes sobre dichos asuntos.

\section{Abstract}

This paper shows the results of a study carried out with young people in Caldas State, Colombia. According to the DANE (2005), the youth population estimated for 2007 was of 237,130, that is, 97,789 between 14 and 18 years; 74,413 between 19 and 22 years and 64,928 between 23 and 26 years. In this study, the probabilistic sample was of 4,479 young people and the surveyed population was of 4,559 young people. They belonged to the 27 municipalities of Caldas and were determined proportionally based on the population of each municipality.

This study allows to evidence the thinking of young people, their interests, motivations, and viewpoints related to politics, their perception of the country, and political parties and movements. It also analyzes the level of information about laws and institutions related to these people and the opportunities young people can get from them.

The development of each subtopic is distributed based on: 1) age and gender, 2) subregions, and 3) occupations or professions and geographical location. This information allows a first description and, from which, some conceptual approaches to the topics are proposed. Finally, general conclusions derived from the information given by young people are drawn.

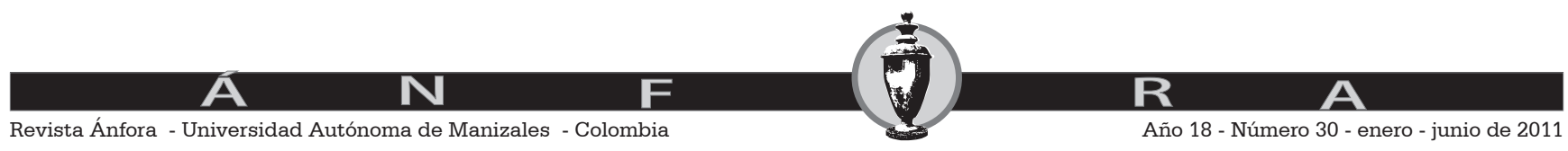




\section{Introducción}

Para dar cumplimiento al artículo 45 de la Constitución Política de Colombia de 1991 y a la Ley 375 del 4 de julio de 1997 (Ley de la Juventud Colombiana), la gobernación del Departamento de Caldas expidió el Decreto No. 0654 en 2007, mediante el cual adoptó la política pública de juventud. En esta política se establecen once áreas: trabajo, salud, comunicación, educación, economía, deporte, recreación, cultura, familia, medio ambiente, la participación y política.

La investigación en general, buscaba identificar las características de los jóvenes del Departamento de Caldas con respecto de cada uno de los tópicos mencionados. Ello abarca a todos los jóvenes, puesto que se hizo con base en una muestra probabilística estratificada (4.479, y los consultados 4.559), según cada una de las áreas de la política pública, pero este artículo se centra en el área política y de la participación. Para el acceso a estos dos ámbitos se asume conceptualmente que los jóvenes son sujetos autónomos, que pueden ejercer como interlocutores válidos y conscientes, cuando se trata de la reflexión, discusión y definición de sus puntos de vista acerca de los asuntos públicos.

La investigación es descriptiva, dado que intenta evidenciar las características que manifiestan los jóvenes en las diferentes áreas de la política pública y se respalda en un cuestionario que se elaboró a partir de las encuestas nacionales que aplican los Observatorios de la Juventud de España, México y de Chile. Este instrumento tuvo la validación de pares externos de España y de Chile, y de académicos de Colombia vinculados a este tipo de estudios. Con esto, en nuestro caso específico, se buscaba identificar lo que piensan y les llama la atención a los jóvenes sobre los asuntos de la política y de la participación, tal como ellos la entienden y como la asumen,(sin otras inferencias al respecto), de sus intereses, motivaciones, se les consulta acerca de los puntos de vista en relación con la situación del país, lo que piensan de los partidos y movimientos políticos, el nivel de información que tienen acerca de las normas e instituciones relacionadas con ellos, y de las posibilidades y oportunidades que ellas les brindan.

Los resultados se elaboran teniendo como base las tabulaciones que aparecieron luego de usar el programa de estadísticas SPSS (Statistical Package for the Social Sciences), desde allí se muestra lo que estos jóvenes expresan, incluyendo sus nociones y prenociones sobre el tema, haciendo uso de los cuadros y gráficas que surgen de la tabulación de los datos, por tanto, no se apoyan en hipótesis previas, sólo evidencian sus expresiones, sin embargo, las interpretaciones se respaldan en los planteamientos teóricos de algunos autores que se refieren a estos asuntos. En principio se les indaga por la magnitud de su interés por la política.

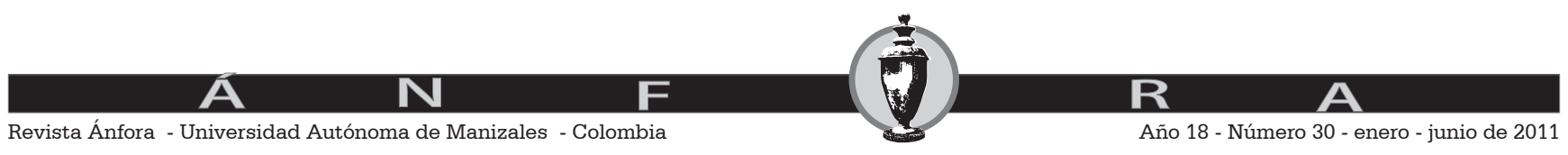




\section{Interés por la política}

La Comisión Económica para América Latina -CEPAL- y la Organización Iberomericana de Juventud -OIJ-, (2007), plantean que la participación social y política es un aspecto determinante para la inclusión de los jóvenes. A través de ellas, se hacen explícitos sus intereses, compromisos y responsabilidades con la construcción de la sociedad. En sentido similar, la Constitución Política de Colombia de 1991, indica que la participación política se respalda en el uso que los ciudadanos hacen de los mecanismos establecidos para que intervengan en la conformación, ejercicio y control del poder público. Su ejercicio se ampara en los derechos políticos, tanto individuales como colectivos que son el sustento de la democracia participativa.

El ámbito de lo que significa político se adopta de Zemmelman (2001: 37), cuando dice que "lo político constituye una forma de pensar la realidad histórica según el modo como se ha ido estructurando la conciencia social, antes que desde cómo se muestra la morfología de la realidad social". En ese sentido este escrito explora, en principio, la magnitud del interés que manifiestan los jóvenes del Departamento de Caldas con relación a su participación en asuntos de la política.
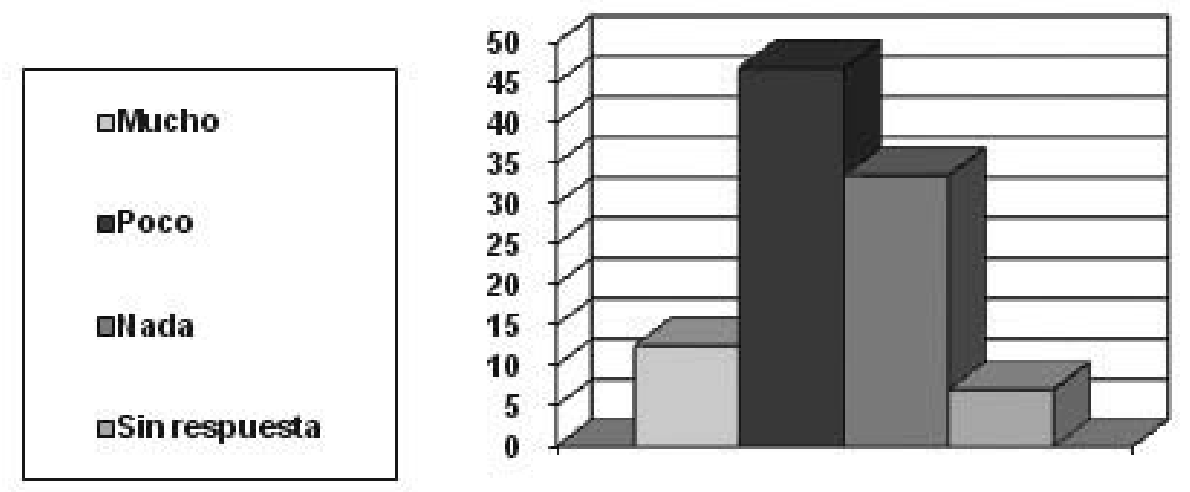

Gráfico 1. Interés de los jóvenes por la política. (\%)

En el gráfico 1 se observa que el interés de los jóvenes de Caldas por la política, es muy bajo, el 80,5\% dice interesarse poco o nada por estos asuntos. Esta cifra puede aumentar si se tiene en cuenta que $7,1 \%$ no responde.

Aunque diversos organismos internacionales expresan cada vez más la importancia de que se estimule la inclusión de los jóvenes en la vida de las sociedades, éstos expresan reiteradamente su desinterés por los asuntos de la política, al respecto, podría pensarse que se hace mención de la política tradicional, dado que por ahora, desde esta encuesta, no se generan indagaciones sobre otras posibilidades de pensar la política. Por lo visto, al menos desde las formas tradicionales de hacer la política, a los jóvenes no les gusta la manera como funcionan las instituciones públicas en relación con los asuntos de la política. Al respecto CEPAL (2003: 11 - 12) plantea:

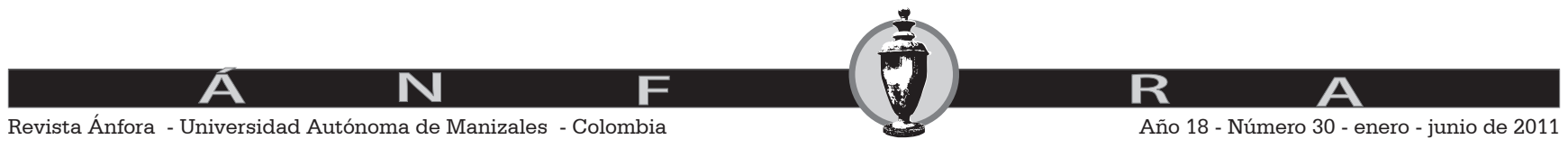


En verdad, todo parece indicar que la real o supuesta apatía juvenil se relaciona con el desencanto que estarían produciendo instituciones que funcionan cada vez más en el marco de rutinas bastante más aburridas que espectaculares en materia de innovaciones... lo que contrasta con la mentalidad predominante en los jóvenes que quisieran ser testigos de cambios rápidos y de fondo en muy diversas esferas del funcionamiento societal.

Agrega la CEPAL que en las últimas décadas, la juventud evidencia que sus vínculos con la política son débiles, así como con la transformación y con el cambio social, dado que su participación se hace en ámbitos donde predomina lo local, y las actividades que realizan, circulan por caminos diferentes a los que proponen los partidos políticos (CEPAL-OIJ, 2007). Además, sienten que la sociedad les brinda oportunidades muy precarias y que les restringe su participación en la política y el ejercicio de derechos sociales. Por otra parte, los consumos culturales y las redes virtuales, entre otras cosas, inciden en que los jóvenes construyan otros intereses con respecto de su participación social.

En relación con el género, la tendencia general se mantiene. Tanto los hombres (77\%) como las mujeres (84\%) tienen poco o ningún interés por la política. Sin embargo, los hombres expresan un mayor interés por la política y son más los que no responden sobre este aspecto. Las mujeres, en cambio, muestran menor interés por estos asuntos y son menos las que dejan sin respuesta esta pregunta.

Al compararlos por grupos de edad la tendencia es similar, siendo un poco más bajo el desinterés en los jóvenes entre 19 y 22 años (77\%). En la siguiente tabla se puede percibir el interés de los jóvenes por la política, teniendo como base la ubicación geográfica. Veamos los resultados.

\begin{tabular}{|l|r|r|r|}
\hline \multicolumn{1}{|c|}{ Sub región } & \multicolumn{1}{c|}{ Mucho } & Poco o nada & No responde \\
\hline Centro Sur & 13 & 78 & 9 \\
\hline Norte & 9 & 88 & 3 \\
\hline Occidente Alto & 11 & 86 & 3 \\
\hline Occidente bajo & 12 & 84 & 4 \\
\hline Magdalena Caldense & 16 & 76 & 8 \\
\hline Alto Oriente & 11 & 82 & 7 \\
\hline
\end{tabular}

Tabla 1. Interés de los jóvenes por la política, discriminado por ubicación geográfica (\%)

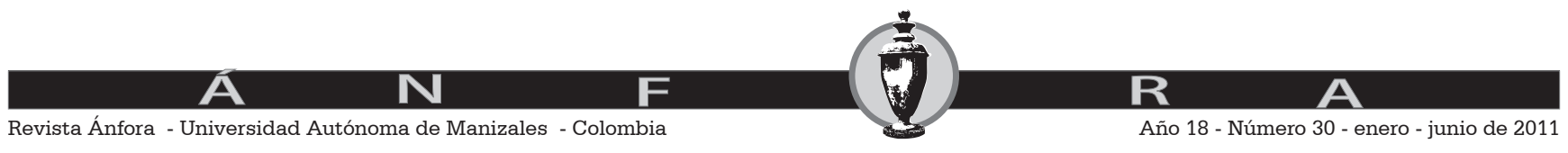


Con respecto a la ubicación geográfica, el poco o ningún interés por la política se ubica entre el $82 \%$ y el $88 \%$, excepto para los jóvenes de las regiones Centro Sur y Magdalena Caldense que manifiestan un desinterés del 78\% y 76\% respectivamente.

Si se comparan los resultados de la encuesta sobre cultura política realizada a mayores de 18 años para Colombia (DANE, 2007), se puede observar que en el Departamento de Caldas es notorio el desinterés por la política a nivel de los jóvenes, dado que dicho estudio muestra que la cuarta parte de los colombianos $24,85 \%$ se interesa mucho por los temas de la política, poco el $31,08 \%$, y nada el 14,57\%, los demás no responden.

Otro aspecto que merece nuestra atención tiene que ver con las actividades que realizan los jóvenes del departamento, en el sentido de mirar lo que piensan sobre este asunto los que trabajan, estudian, realizan ambas actividades o en su defecto ninguna de ellas. Veamos los resultados:

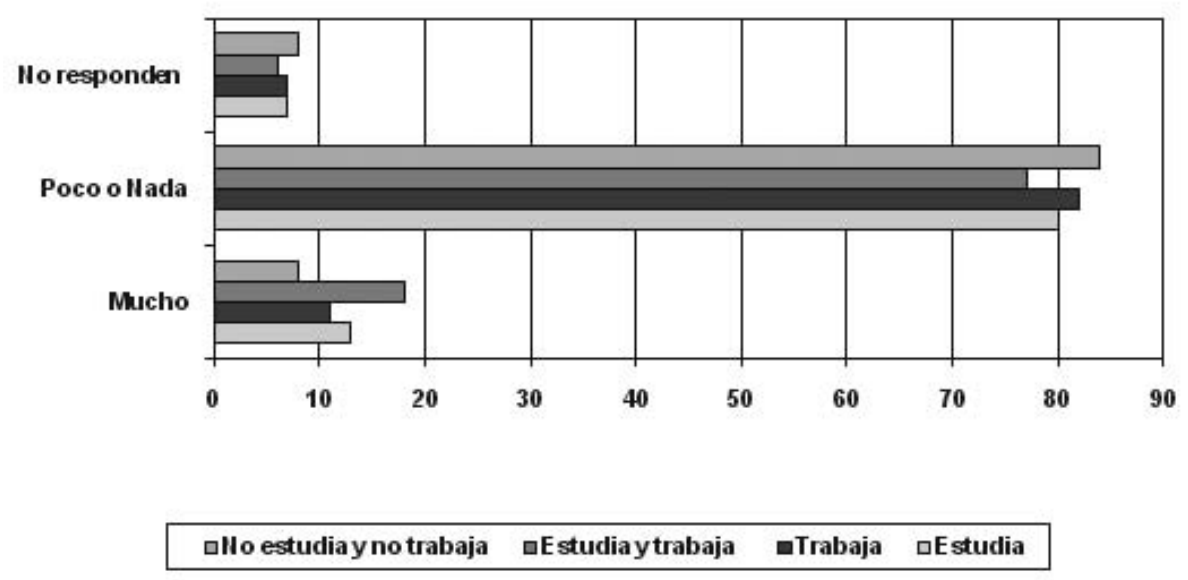

Gráfico 2. Interés de los jóvenes por la política, discriminado por actividad (\%)

Si bien la tendencia de los datos se mantiene, es mayor el interés por la política de los jóvenes que estudian y trabajan, con respecto de los que sólo estudian, o únicamente trabajan, o no hacen ninguna de las dos actividades. El grupo menos preocupado por la política es es de los que no trabajan ni estudian $84 \%$ quienes responden poco o nada.

Si miramos el interés de los jóvenes por la política, según su ubicación geográfica, se puede evidenciar que hay mayor desinterés en los jóvenes rurales $85 \%$, que en los urbanos $77 \%$. Se destaca el porcentaje de jóvenes urbanos que expresó tener mucho interés por la política 15\%, en comparación con los jóvenes rurales $9 \%$. Es claro que son más las posibilidades de ocupar un espacio en lo público para los jóvenes de las zonas urbanas que para los de las zonas rurales, la exclusión, las restricciones de acceso, la desinformación y las difíciles condiciones de vida de las personas del campo pueden incidir en esta tendencia.

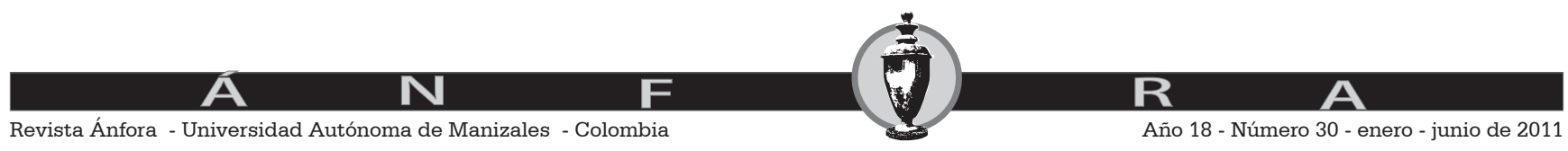


En general, alrededor de tres cuartas partes de los jóvenes expresan su poco o ningún interés por la política, mientras un poco menos de la sexta parte manifiesta que sí les interesa mucho estos asuntos. Es marcado el contraste entre una y otra posición, de ahí que se requiere de una indagación más profunda.

\section{Puntos de vista sobre la situación política del país}

Cuando los jóvenes opinan sobre la situación política del país muestran algunas paradojas, ello puede evidenciarse en los resultados de la siguiente tabla.

\begin{tabular}{|l|r|r|r|r|}
\hline \multicolumn{1}{|c|}{ Afirmaciones } & \multicolumn{1}{c|}{$\begin{array}{c}\text { De } \\
\text { acuerdo }\end{array}$} & En desacuerdo & No saben & No responden \\
\hline Colombia es un país democrático & 55 & 26 & 14 & 5 \\
\hline Se respetan los derechos humanos & 29 & 56 & 10 & 6 \\
\hline Es un país con gobierno autoritario & 42 & 33 & 19 & 6 \\
\hline $\begin{array}{l}\text { La gente tiene posibilidad de expresarse y } \\
\text { opinar }\end{array}$ & 41 & 41 & 12 & 6 \\
\hline Hay diferencias sociales y desigualdad & 58 & 24 & 11 & 6 \\
\hline $\begin{array}{l}\text { La forma de elegir los gobernantes es trans- } \\
\text { parente }\end{array}$ & 27 & 47 & 18 & 7 \\
\hline $\begin{array}{l}\text { Los jóvenes son escuchados y tenidos en } \\
\text { cuenta }\end{array}$ & 22 & 56 & 17 & 6 \\
\hline $\begin{array}{l}\text { A los ciudadanos les interesan los asuntos } \\
\text { políticos }\end{array}$ & 35 & 36 & 24 & 6 \\
\hline $\begin{array}{l}\text { Hay posibilidades de participación para los } \\
\text { jóvenes }\end{array}$ & 40 & 37 & 19 & 6 \\
\hline Hay corrupción & 63 & 15 & 15 & 7 \\
\hline
\end{tabular}

Tabla 2. Pensamientos acerca de la situación política de Colombia (\%)

Tal como puede observarse en la tabla 2, los jóvenes consideran que Colombia es un país democrático 55\%. Sin embargo, es una democracia sui generis porque el 56\% está en desacuerdo con la afirmación de que en el país se respetan los derechos humanos, mientras el $47 \%$ opina que la forma de elegir a los gobernantes no es transparente.

Esto cuestiona a una sociedad definida como un Estado Social de Derecho donde se deben privilegiar los derechos de los ciudadanos, igualmente interroga al Estado democrático, ente del que se espera surjan decisiones colectivas a través de mecanismos que garanticen la participación de la población en condiciones de equidad y de respeto por las reglas de juego. Ello evidencia un contrasentido cuando afirman de que viven en un país democrático, y en

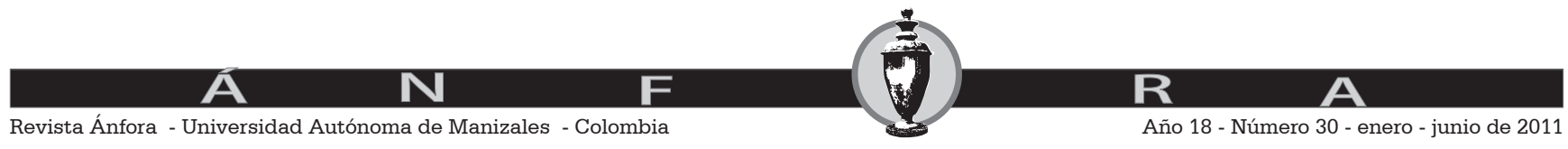


la siguiente pregunta, responden que "los derechos humanos no se respetan", cuando es una de las condiciones elementales de la democracia moderna. Además, estos jóvenes opinan que en el país hay marcadas diferencias sociales $58 \%$, alta tasa de corrupción $63 \%$, es un país con un gobierno autoritario $42 \%$ y $56 \%$ piensan que no los escuchan ni los tienen en cuenta como deliberantes ni como decisores. Al parecer aprenden a convivir con los vicios que acompañan las circunstancias en las cuales se ejerce la democracia.

Se percibe distancia entre los intereses personales particulares y los asuntos de la política, dado que afirman que "no se sienten representados", e indican "no voy a participar porque mi voto no cuenta" o consideran que "siempre sucede lo mismo". Hay desconfianza frente el funcionamiento de las instituciones democráticas, lo cual genera prácticas políticas que los lleva a marginarse y despreciar las formas hegemónicas de orientar la sociedad, y optan por resolver su socialidad personal, acercándose cada vez más a lo particular y a lo privado.

Al contrastar esta información, por género, edad, subregiones, actividad y ubicación geográfica se confirman las tendencias generales con algunas particularidades.

Con respecto a la edad y el género, más de la mitad de los jóvenes confirma los hallazgos iniciales, según los cuales Colombia es un país democrático, los que más aceptan este punto de vista son los de 14 a 18 años 60\%. Los demás se encuentran a más de 5 puntos porcentuales por debajo. Quienes más están en desacuerdo con esta afirmación son los jóvenes mayores de 23 años 30\%.

Son más los jóvenes que están en desacuerdo con que en este país se respetan los derechos humanos, sobre todo los jóvenes mayores de 23 años $61 \%$. En contraste, quienes están de acuerdo con que sí se respetan los derechos humanos no llegan a la tercera parte de los jóvenes consultados. La comparación por género, si bien no indica diferencias significativas, muestra que son más las mujeres que los hombres con sólo unos puntos de diferencia, quienes manifiestan su desacuerdo con esta opinión.

Son más las personas que consideran que en Colombia hay diferencias sociales y desigualdad. Estos puntos de vista son aceptados en porcentajes que van desde $57 \%$ a $61 \%$. Menos de la tercera parte de los jóvenes está de acuerdo en que la forma de elegir a los gobernantes es transparente, mientras que cerca del $50 \%$ piensa lo contrario. Se diferencian un poco los más jóvenes, entre 14 y 18 años, que presentan un desacuerdo menor con esta opinión 43\%, en comparación con los otros grupos de edad.

Respecto a la corrupción, los porcentajes de aceptación son superiores al 63\%, salvo en el caso de los más jóvenes lo expresan con el 59\%. En general, los hombres y las mujeres coinciden en que hay altos índices de corrupción.

De SOUZA Santos (2006, cap3: 5) indica que la corrupción se naturaliza y ello puede convertirse en una estrategia de los sectores que controlan el poder
4 DE SOUZA Santos, Boaventura. Para una democracia de alta intensidad, Capítulo III del texto Rein-ventar la emancipación social. Buenos Aires: Clacso, 2006, pág 5 .

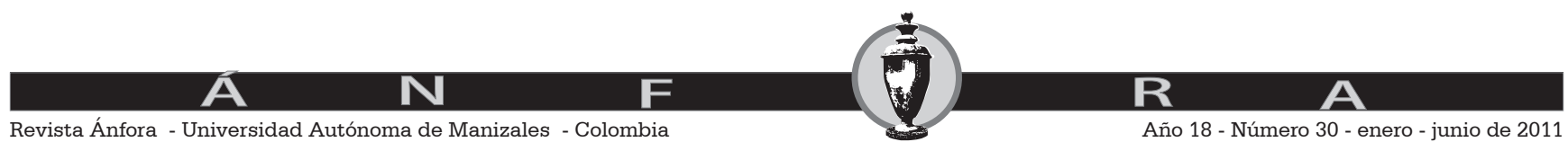


político, dado que contribuyen a generar y a mantener una democracia de "baja intensidad", en donde se "naturaliza la distancia de los ciudadanos con respecto de la política": puesto que es común escucharlos decir que "todos son corruptos", "los políticos son todos iguales", etc., lo cual es funcional al sistema político para mantener a los ciudadanos apartados. Por ello, la naturalización de la corrupción es un aspecto fundamental de este proceso".

Con respecto a sí Colombia es un país democrático, en las subregiones se observa que mientras los jóvenes de todas las regiones manifiestan su acuerdo con esta opinión en porcentajes entre el 56\% y 65\%, la región de Centro sur, que concentra el mayor número de jóvenes del departamento, manifiesta un acuerdo del $49 \%$ y un desacuerdo del 31\%. Esta región es la que más desacuerdo expresa sobre si en Colombia se respetan los derechos humanos 59\%, mientras, Occidente Bajo presenta mayores índices de aceptación 37\%.

Son muchos más los jóvenes que plantean su desacuerdo con la afirmación de que la elección de los gobernantes es transparente. En todos los casos, es mayoritario el punto de vista que sostiene su desacuerdo al respecto. Los de Occidente Bajo muestran un mayor contraste entre ambas opciones 35\% de acuerdo y el $38 \%$ en desacuerdo. Esta región es la que más contrasta con Centro Sur que tiene un desacuerdo de $50 \%$ y un acuerdo de $25 \%$.

Hay diferencia notable cuando se afirma que los gobernantes atienden y escuchan las necesidades de los jóvenes, puesto que en la subregión Occidente Bajo 47\% dicen que están en desacuerdo, las demás subregiones evidencian ese desacuerdo en $57 \%$. De manera similar, expresan que no son escuchados $53 \%$, salvo en el caso de esa subregión $49 \%$.

En la subregión Centro Sur es donde menos se considera que los ciudadanos se interesan por los asuntos políticos $31 \%$ y es donde expresan mayor desacuerdo con esa afirmación $41 \%$. En esa subregión se plantea mayor desacuerdo con que existen posibilidades para que los jóvenes participen. En contraste sobre este aspecto, en las demás subregiones se indica de manera mayoritaria su acuerdo con que sí hay posibilidades de participación para los jóvenes, cifras que rondan el $40 \%$. En promedio, alrededor de la cuarta parte de los consultados dice no saber sobre el asunto. Da la impresión de que desconocen o no les interesan las posibilidades de participar en la sociedad desde su condición de jóvenes.

En relación con la apreciación de que Colombia es un país democrático, los jóvenes que estudian y los que viven en zonas rurales son los que más están de acuerdo con la afirmación $57 \%$ y $58 \%$ respectivamente, mientras que los que no estudian ni trabajan, presentan menor porcentaje de estar de acuerdo $45 \%$.

Mediante consideraciones que oscilan entre 58\% y 59\%, los jóvenes que no estudian ni trabajan y los que trabajan, expresan mayor desacuerdo con la afirmación de que en este país se respetan los derechos humanos. De manera similar, los jóvenes urbanos $60 \%$ y los rurales $51 \%$ están de acuerdo con este punto de vista.

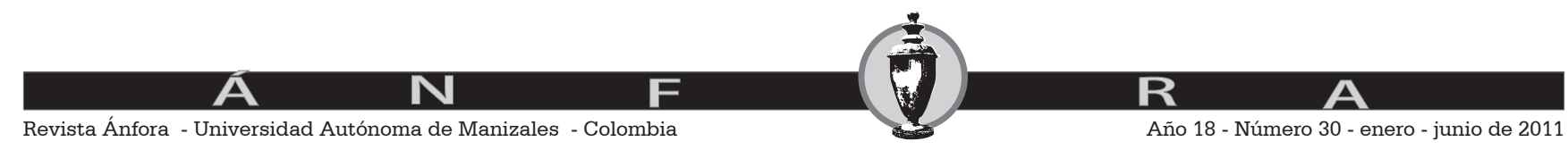


En general, los jóvenes están en desacuerdo con que son escuchados y tenidos en cuenta, con valores que oscilan entre $50 \%$ y $60 \%$, expresando los niveles más altos los de la zona urbana con el $60 \%$ mientras que en el extremo inferior están los de la zona rural, que se equiparan con los que no estudian ni trabajan, esto con el $50 \%$.

Los jóvenes, independientemente de la actividad que desarrollan y de la ubicación, coinciden en que es notoria la corrupción. Los niveles de aceptación de este planteamiento superen el $60 \%$ y llegan hasta $66 \%$, estando en el rango inferior los jóvenes que viven en la zona rural.

\section{Pensamientos sobre los partidos y movimientos políticos}

A continuación se presentan los resultados de la indagación respecto lo que piensan los jóvenes de Caldas sobre los movimientos y partidos políticos.

\begin{tabular}{|l|r|r|r|r|}
\hline Afirmaciones & $\begin{array}{l}\text { De } \\
\text { acuerdo }\end{array}$ & En desacuerdo & No saben & No responden \\
\hline Los políticos se preocupan por los jóvenes & 18 & 56 & 21 & 4 \\
\hline $\begin{array}{l}\text { Los partidos políticos representan las in- } \\
\text { quietudes de los jóvenes }\end{array}$ & 17 & 56 & 23 & 4 \\
\hline $\begin{array}{l}\text { Los partidos políticos priorizan el trabajo } \\
\text { con jóvenes }\end{array}$ & 17 & 53 & 25 & 5 \\
\hline La política se hace solo desde los partidos & 37 & 28 & 28 & 6 \\
\hline $\begin{array}{l}\text { Los partidos y movimientos políticos tienen } \\
\text { espacios de participación para los jóvenes }\end{array}$ & 30 & 39 & 27 & 5 \\
\hline $\begin{array}{l}\text { Los jóvenes aspiran a cargos públicos apoya- } \\
\text { dos por los partidos y movimientos políticos }\end{array}$ & 40 & 28 & 27 & 5 \\
\hline
\end{tabular}

Tabla 3. Puntos de vista sobre los partidos y movimientos políticos (\%)

Como puede observarse en la tabla 3, es amplia la cantidad de consultados que considera que los movimientos políticos, los políticos y los partidos que ellos representan, se han despreocupado por los asuntos de los jóvenes. Se percibe un notorio desplazamiento de la razón de ser de la política como construcción de voluntades, al de la política pensada como una actividad exclusiva de los partidos políticos. Parece sugerirse la existencia de un ámbito de la vida orientado a la política, donde hay actores especializados en ella que la asume como un campo de desempeño exclusivo. El asunto es que estos jóvenes asumen que los partidos "no les ofrecen espacios de participación" 39\%, aunque expresan que tienen la ilusión de acceder a cargos públicos apoyados en el respaldo que les puedan ofrecer los diferentes movimientos políticos 40\%, y consideran que "la política se hace sólo desde los partidos" 37\%.

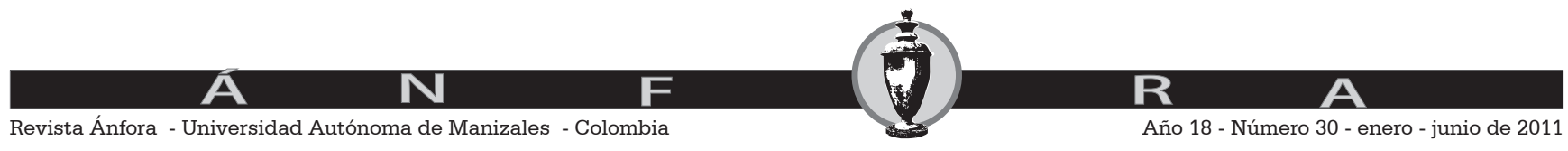


Si se tiene en cuenta que una función importante de los partidos políticos es facilitar la participación de los ciudadanos, involucrarlos en los asuntos del manejo del poder público y fungir como los mediadores entre la sociedad y el Estado, según lo expresado por los jóvenes, en el país hay una seria deuda de estas organizaciones sobre el reconocimiento de las nuevas generaciones de ciudadanos. Los partidos no ejercen como organizaciones que recojan la pluralidad de ideas e intereses de los jóvenes del país. Esto muestra distancia entre los partidos políticos y los jóvenes, en otras palabras, se puede expresar que no existe, o es inadecuada la articulación entre las redes sociales juveniles y los partidos políticos, por lo cual son pocos los elementos que permiten cohesionar los deseos y las aspiraciones de los jóvenes con los lineamientos y plataformas ideológicas de los partidos.

Si se considera que los partidos políticos son entes que contribuyen a la producción de las subjetividades que deambulan en los colectivos sobre los asuntos de la política, de alguna manera han tenido la responsabilidad de cimentar las barreras, imaginarias o reales, que permiten o impiden el ejercicio de la participación política. Se trata de no olvidar que los partidos obstaculizan o fomentan el interés por la práctica política relacionada con la vida colectiva.

Hay crisis en los partidos cuando se pierde la credibilidad en ellos, dado su ejercicio político. La poca empatía con ellos se sustenta en la no representación efectiva de la pluralidad de intereses y necesidades de la población. Los partidos carecen de la posibilidad de ser vehículos de participación real de la gente en los asuntos que le afectan y, por el contrario, dan la sensación de que son poco útiles cuando se trata de atender las demandas de la población. $\mathrm{Al}$ respecto De Souza Santos (2006: 8) expresa su perspectiva sobre el papel que cumplen los partidos políticos en la actualidad:

“los partidos políticos están perdiendo el control de la agenda política: nunca incumplieron tanto sus promesas electorales cuando llegaron al poder como en estos momentos. Uno de los estudios más interesantes es mirar los programas de los partidos y después su práctica política. Ha sido siempre así, pero ahora todavía más, porque hay una presión de la globalización neoliberal que no puede entrar en la agenda política de un partido. Ninguno de ellos puede decir "cuando llegue al poder voy a seguir totalmente las instrucciones del BM y del FMI", porque si dice esto no va a tener votos, ya que la gente sabe las consecuencias de eso. Tiene que decir que va a dar más empleo, educación, salud, etc., pero cuando llega al poder no hace nada de eso. Este incumplimiento hace que la deslegitimación de los partidos sea cada vez más grande en un número cada vez mayor de países". 
Como en los casos anteriores, el análisis de esta información se discrimina en tres grupos: el género y la edad, las subregiones y las actividades que realizan según la ubicación geográfica.

En los grupos de edad y género se perciben algunas diferencias. La opinión de que "los políticos se preocupan por los jóvenes" es más cuestionada por el grupo de 23 a 26 años, 58\%. La afirmación de que "los partidos políticos representan las inquietudes de los jóvenes", muestra mayor desacuerdo en los hombres $58 \%$ que en las mujeres $54 \%$.

A medida que aumenta la edad de los jóvenes se acepta la afirmación de que "la política se hace sólo desde los partidos", pasa del 33\% a 37\% y 41\% según cada grupo de edad establecido para este estudio, al parecer su experiencia de vida los lleva a reconocer que las prácticas políticas en esta sociedad funcionan de esa manera. Con referencia a si "los partidos y movimientos políticos tienen espacios de participación para los jóvenes", son un poco más críticos los hombres que las mujeres $40 \%$ y $30 \%$ respectivamente. Con respecto a la afirmación de que "los jóvenes aspiran a cargos públicos apoyados por los partidos y movimientos políticos", de nuevo el aumento progresivo en la edad muestra que las prácticas políticas los llevan a requerir de los grupos políticos para poder aspirar a ingresar al campo laboral, pasando progresivamente del $37 \%$, a $40 \%$ y $42 \%$, según los grupos de edad que hemos definido.

En este sentido, de acuerdo con la subregión de donde proceden, los jóvenes del Norte y de Alto oriente se desatacan porque casi la mitad de ellos considera que para aspirar a cargos públicos deben apoyarse en los partidos y movimientos políticos, en porcentajes de $47 \%$ y $49 \%$ respectivamente, mientras que los demás se encuentran cerca del $40 \%$.

En relación con la actividad que realizan y a su ubicación geográfica, los jóvenes de la zona urbana son quienes más están en desacuerdo con la afirmación de que "los políticos se preocupan por los jóvenes" 59\%. Mientras la opinión de que "los partidos políticos representan las inquietudes de los jóvenes", es compartida por aquellos que sólo estudian o estudian y trabajan, con cifras cercanas a la quinta parte de los consultados, mientras que los que menos aceptan esa afirmación son los que no estudian ni trabajan, con el 15\%, evidenciando que pueden sentirse en situación de exclusión social. En este mismo sentido, la opinión sobre que "los jóvenes que aspiran a cargos públicos apoyados por los partidos y movimientos políticos", es más aceptada por los que estudian y trabajan $46 \%$, evidenciando que su actividad laboral pudo ser consecuencia de este hecho.

Al indagarse por la identificación de los jóvenes con respecto de cada uno de los partidos o movimientos políticos, se pudo observar que frente al grado de conocimiento, filiación y compenetración con los partidos políticos, los resultados señalan que los partidos menos conocidos por los jóvenes de Caldas son los de oposición, como el Movimiento Obrero Independiente Revolucionario -MOIR-35,3\%, el partido Comunista 31,8\% y la Alianza Social Indígena 31,8\%.

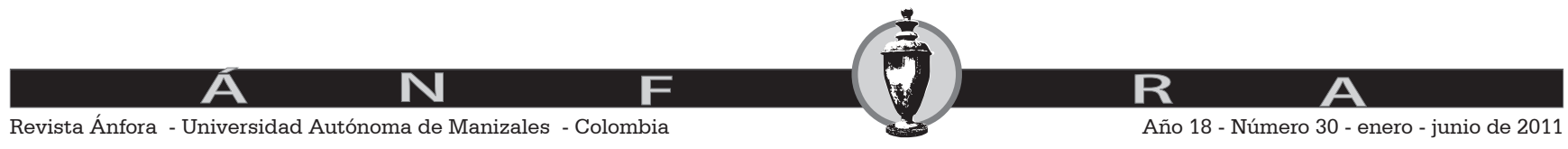


Por el contrario, son más conocidos los partidos y movimientos políticos que hacen parte del oficialismo, tales como el partido liberal, el conservador y la Unidad de Salvación Nacional, o partido de la "U", entre otros.

En esta misma línea, las ideas que más les gustan a los jóvenes tienen que ver con los partidos tradicionales como el liberal $11,2 \%$, el conservador $9,2 \%$ y el del partido de la U 12,2\%, sin que lleguen a ser significativos los porcentajes, pues apenas superan el 10\% de los encuEstados.

El sondeo por la confianza en los líderes de estos partidos y la acción de votar por ellos muestra porcentajes bajos de respuestas $6,4 \%$, mientras la consulta por el desinterés en los partidos presenta un promedio de $48,3 \%$ de respuesta. Cerca de la mitad de los jóvenes considera que no les interesa los diferentes grupos políticos, situación que podría mostrar que más que apoyar los grupos y sus programas, sus posibles respaldos políticos tendrían que ver con las maneras como se ejerce la política y los lideres que emerjan en cada coyuntura. Antes que adscripciones racionales, estaríamos frente a apoyos a la significatividad de las propuestas, de otra forma adoptarían posturas de marginación.

El hecho de que la mayoría de los jóvenes de Caldas no muestra filiación ni interés por los partidos políticos, confirma su distancia frente a las instituciones y actores políticos, y la percepción de que sus demandas no son consideradas, ni representadas por ellos, conclusión similar a la de estudios como el de la CEPAL-OIJ (2007) y el de la CEPAL, AECID, UN, OIJ (2008).

Al contrastar por grupos de edad, se observa que a menor edad de los jóvenes más desconocen los partidos o movimientos políticos y a mayor edad tienen más conocimiento, sin que la información política sea suficiente, puesto que al expresar el gusto por las ideas de los partidos y la confianza en los líderes, muestran los resultados que se encuentran por debajo de $10 \%$.

También se observa que el Centro Sur y en Alto Oriente, son las subregiones donde más se desconocen los partidos tradicionales Liberal y Conservador, en un promedio de 24,5\% para el Conservador y 28,5\% para el Liberal. Algo similar ocurre con Cambio Radical 28\%. El partido de la U es el más conocido de los que hacen parte de la Coalición de Gobierno en el país. Este último es desconocido por la quinta parte de los jóvenes.

Con respecto a los grupos de oposición, los menos conocidos son el MOIR, el Partido Comunista y la Alianza Social Indígena, con promedios que corresponden a cerca de la tercera parte de los jóvenes, mientras que el más conocido de estos grupos políticos es el Polo Democrático, con cerca de un poco más de la quinta parte de los consultados que lo desconocen, en contraste con las regiones Norte y Occidente Bajo donde menos interés tienen los jóvenes por el Polo Democrático, con el 63\%. Al partido de la U donde más lo desconocen es en la región Centro Sur con 22\%, y en la región de Alto Oriente 19\%.

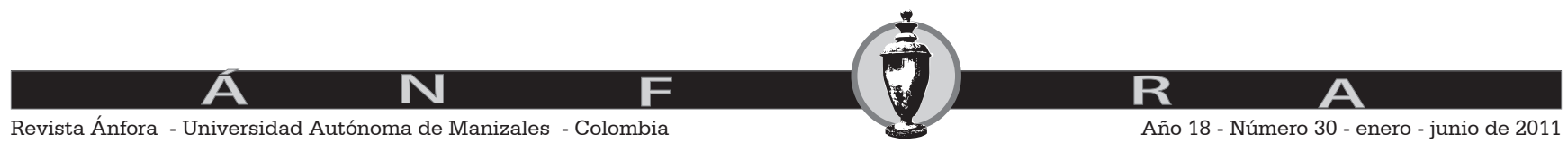


Los mayores porcentajes que expresan los jóvenes de las diferentes subregiones, están orientados a mostrar un desinterés por los distintos partidos, al decir "no les interesa" unos más que otros, pero con afirmaciones contundentes que superan la mitad de los jóvenes del departamento.

Así mismo, la comparación por ubicación geográfica y por quienes estudian o trabajan, no muestra diferencias notables en las opiniones de los jóvenes cuando se refieren a los partidos políticos. Sin embargo, los índices dependen de que sean los partidos tradicionales que hacen parte de la coalición de gobierno o de la oposición. El desconocimiento de los primeros es un poco más de la quinta parte, mientras que en los segundos es cerca de la tercera parte.

Los que no estudian ni trabajan son quienes presentan los mayores porcentajes de desinterés por los partidos políticos, en muchos casos superando la mitad de los jóvenes consultados. Son notables los resultados en el sentido de evidenciar los índices inferiores al 10\% cuando se les pregunta acerca del gusto por las ideas de los partidos, la confianza en los líderes y en el interés de votar por ellos, dado que en estos últimos casos incluso están por debajo del 5\%. Salvo en el partido de la U. donde hay un poco de mayor aceptación, puesto que algunos ítems superan el $10 \%$.

\section{Participación en las elecciones}

Usualmente se ha asumido la democracia como una forma de gobierno que se instaura en la medida en que los gobernantes son electos en comicios libres, es decir, cuando los gobernados intervienen en la toma de decisiones. En la actualidad, se diferencia la democracia representativa de la democracia participativa. En la primera, predomina la delegación del poder por parte del pueblo en quienes los representan. En la segunda, los ciudadanos se vinculan a la dirección de la sociedad mediante mecanismos diferentes del voto y son partícipes de las decisiones. En Colombia, los mecanismos consagrados en el artículo 103 de la Constitución Política y reglamentados en la Ley 134 de 1994 son la consulta popular, el referendo, el plebiscito, la iniciativa popular legislativa, el cabildo abierto y la revocatoria del mandato.

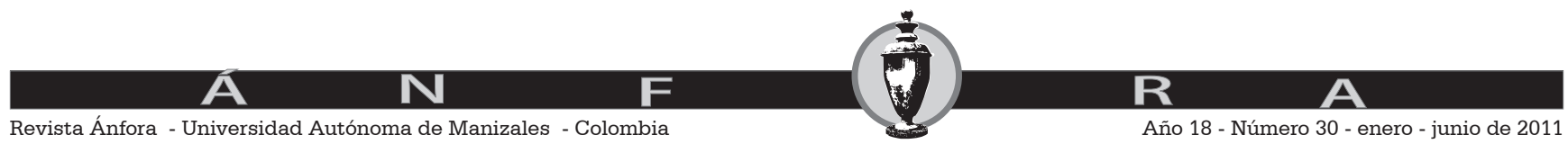




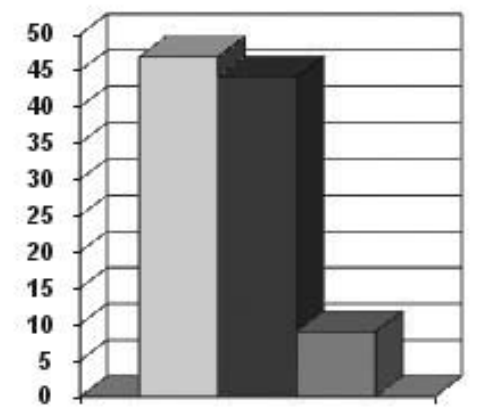

Gráfico 3. Participación de los jóvenes del Departamento de Caldas en elecciones. (\%)

La pregunta se refiere al uso que han hecho los jóvenes del derecho al sufragio con respecto de haber votado en cualquier escenario. Se observa que 46,9 $\%$ de los jóvenes ha participado alguna vez en elecciones, mientras $44 \%$ expresa que no lo ha hecho, aunque no se refieren necesariamente a elecciones políticas, pues son diversos los eventos en los cuales han votado: comicios a las alcaldías, gobernaciones y presidenciales, o bien elecciones en el colegio relacionadas con el gobierno escolar. Muchos indican que se trata de un derecho y un deber como ciudadanos. En algunos casos, se expresa que lo hacen porque necesitan empleo, lo cual muestra un voto condicionado por la conveniencia que pone en evidencia prácticas corruptas derivadas del clientelismo.

Cuando los jóvenes expresan las razones por las cuales no han votado, aparece la edad y el hecho de carecer de documento de identidad, como justificación para no participar de los comicios electorales. Exponen además su desinterés por hacerlo porque, según dicen, no quieren apoyar a los corruptos. Muestran con ello el desprecio que les produce la corrupción, con lo cual esbozan una crítica a esa práctica, que hace explícita la necesidad de generar procesos de educación acerca del funcionamiento de los asuntos públicos y de las buenas formas de convivencia, para efectos de que se promueva la participación consciente de los ciudadanos.

Lo anterior, sin duda, le da la razón a los constituyentes de 1991, cuando en el artículo 41 de la Carta Magna propugnan por la importancia de brindar educación a los jóvenes en asuntos de cultura ciudadana. Allí se expresa:

"En todas las instituciones de educación, oficiales o privadas, serán obligatorios el estudio de la Constitución y la Instrucción Cívica. Así mismo, se fomentarán prácticas democráticas para el aprendizaje de los principios y valores de la participación ciudadana" (Artículo 41, Constitución Política de Colombia de 1991).

A continuación se presentan otros aspectos de relevancia, ellos son los relacionados con la discriminación de la participación de los jóvenes en elecciones por grupos de edad, subregiones, actividad de estudiar o trabajar y ubicación geográfica, Veamos:

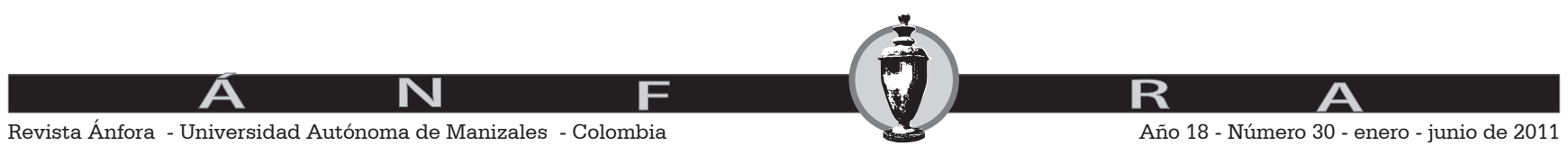




\begin{tabular}{|c|c|c|c|c|}
\hline \multicolumn{2}{|c|}{ Variables } & $\mathbf{S i}$ & No & No responden \\
\hline \multirow{2}{*}{ Género } & Hombre & 46 & 43 & 10 \\
\hline & Mujer & 48 & 45 & 7 \\
\hline \multirow{3}{*}{ Edad } & $14-18$ & 17 & 73 & 10 \\
\hline & $19-22$ & 54 & 37 & 8 \\
\hline & 23- 26 & 71 & 21 & 8 \\
\hline \multirow{6}{*}{ Subregión } & Centro Sur & 48 & 41 & 11 \\
\hline & Norte & 47 & 48 & 5 \\
\hline & Occidente Alto & 47 & 45 & 8 \\
\hline & Occidente bajo & 44 & 50 & 6 \\
\hline & Magdalena Caldense & 44 & 46 & 10 \\
\hline & Alto Oriente & 42 & 51 & 7 \\
\hline \multirow{4}{*}{ Actividad } & Estudia & 37 & 54 & 9 \\
\hline & Trabaja & 66 & 25 & 9 \\
\hline & No estudia ni trabaja & 54 & 37 & 9 \\
\hline & Estudia y trabaja & 60 & 31 & 9 \\
\hline \multirow{2}{*}{ Ubicación } & Urbano & 50 & 40 & 9 \\
\hline & Rural & 43 & 48 & 8 \\
\hline
\end{tabular}

Tabla 4. Participación de los jóvenes del Departamento de Caldas, según el género, la edad, las subregiones, el tipo de actividades que realizan y la ubicación geográfica. (\%).

Como puede apreciarse en la tabla, a medida que aumenta la edad, también se incrementa la participación electoral, siendo ostensible en los jóvenes de 23 a 26 años 71\%. Estos resultados pueden estar relacionados con la existencia de menores oportunidades y espacios de participación para los más jóvenes. Así mismo, la correspondencia entre el aumento de edad y el incremento de participación en elecciones muestra a una mayor cantidad de jóvenes que hacen parte de la toma de decisiones colectivas, no estrictamente políticas, lo que también puede incidir en los resultados.

Si bien por subregiones se mantiene la tendencia general de participar en elecciones, los jóvenes de Centro Sur, Norte y Occidente Bajo son los que más participan. Sin embargo, es interesante ver como alrededor del $45,3 \%$ de los jóvenes ha participado en algún tipo de elección y el $46,8 \%$ no lo hace.

En relación con las actividades de estudiar y trabajar, quienes menos participan son los que sólo estudian con el 54\%, mientras los que sólo trabajan, así como quienes estudian y trabajan al mismo tiempo participan en elecciones con cifras superiores al 60\%. Es posible que el desempeño de actividades que involucran a otros y se realicen en espacios colectivos, tenga alguna relación con estos resultados.

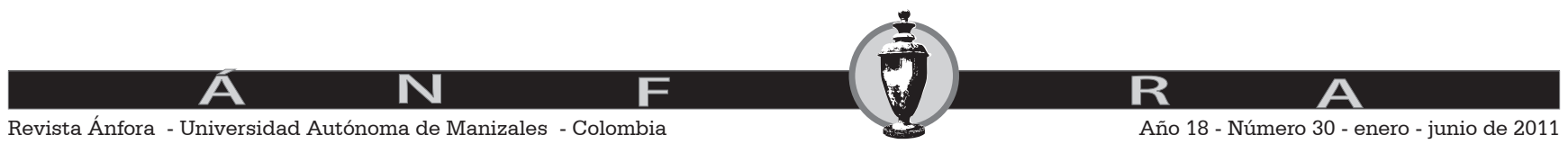


Respecto a la ubicación geográfica, se observa que los jóvenes de la zona urbana participan mayoritariamente, mientras que en la zona rural son más quienes no lo hacen. Sin embargo, los porcentajes de participación en elecciones entre los jóvenes urbanos y rurales son relativamente cercanos, a pesar de las diferencias de sus realidades y la desigualdad de condiciones y oportunidades de participación en las que viven los jóvenes rurales con respecto a los urbanos.

$\mathrm{Al}$ explorar las razones por las cuales los jóvenes han votado en elecciones, lo cual puede observarse en la tabla siguiente, no hubo respuesta de la mayoría que expresó haber votado. Los grupos de edad entre 14 y 18, así como los de 19 a 22 años no expusieron ninguna razón. Las respuestas a este interrogante se organizaron en tres razones expuestas por los jóvenes. La razón más aducida es la de conciencia política, escogida 32,5\%. La segunda razón manifestada son los compromisos y obligaciones adquiridos 11,5\%. La tercera razón expuesta fue la búsqueda de prebendas $7 \%$. La razón que exponen los jóvenes de votar por compromisos y obligaciones adquiridos es muestra de una práctica política que estimula el ir a las urnas como resultado del llamado voto cautivo.

\begin{tabular}{|c|c|c|c|c|c|}
\hline \multicolumn{2}{|c|}{ Variables } & $\begin{array}{l}\text { Buscando } \\
\text { prebendas }\end{array}$ & $\begin{array}{l}\text { Conciencia } \\
\text { política }\end{array}$ & $\begin{array}{c}\text { Compromiso y } \\
\text { obligación }\end{array}$ & $\begin{array}{l}\text { No han votado } \\
\text { y no responden }\end{array}$ \\
\hline \multirow{6}{*}{ 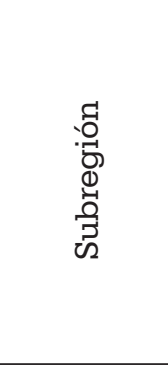 } & Centro Sur & 9 & 37 & 11 & 43 \\
\hline & Norte & 4 & 35 & 17 & 44 \\
\hline & Occidente Alto & 7 & 50 & 15 & 28 \\
\hline & Occidente bajo & 4 & 31 & 16 & 49 \\
\hline & $\begin{array}{l}\text { Magdalena } \\
\text { Caldense }\end{array}$ & 0 & 2 & 0 & 98 \\
\hline & Alto Oriente & 8 & 36 & 12 & 44 \\
\hline \multirow{4}{*}{ 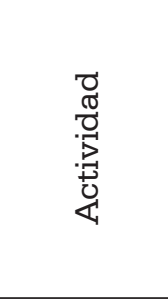 } & Estudia & 7 & 33 & 9 & 51 \\
\hline & Trabaja & 7 & 36 & 14 & 43 \\
\hline & $\begin{array}{l}\text { No estudia ni } \\
\text { trabaja }\end{array}$ & 6 & 40 & 10 & 44 \\
\hline & $\begin{array}{l}\text { Estudia y traba- } \\
\text { ja }\end{array}$ & 8 & 26 & 9 & 57 \\
\hline \multirow{2}{*}{ Ubicación } & Urbano & 7 & 37 & 9 & 47 \\
\hline & Rural & 6 & 31 & 13 & 50 \\
\hline
\end{tabular}

Tabla 5. Razones por las cuales los jóvenes han votado en elecciones (\%).

Los datos por subregión mantienen la tendencia mencionada, sin embargo, se destaca Occidente Alto por ser donde más se inclinan por la conciencia política $50 \%$ como argumento que justifica su acción de votar. Las regiones Occidente Bajo, Occidente Alto y Norte son las que más eligen el compromiso y obligaciones asumida, como razón para votar 17\%. Los jóvenes del Magdalena caldense son los que menos respondieron a esta solicitud $2 \%$.

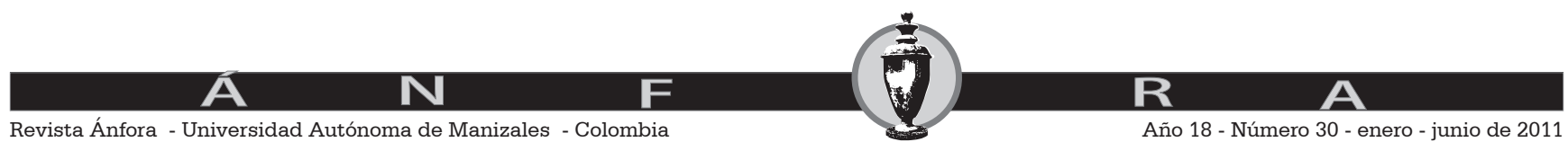


Con respecto a la actividad de estudiar o trabajar, sobresalen los jóvenes que no estudian ni trabajan, por ser quienes en su mayoría expresan que cuando votan lo hacen por conciencia política (40\%).

Igualmente, los jóvenes de la zona urbana (37\%) presentan mayor conciencia política que los de la zona rural (31\%) y, así mismo, en la zona rural son más los que dicen que votan por compromiso y obligación que los de la zona urbana.

\begin{tabular}{|l|r|r|r|}
\hline \multicolumn{1}{|c|}{ Afirmaciones } & De acuerdo & En desacuerdo & No Responden \\
\hline Participar de las elecciones es un derecho & 79 & 15 & 6 \\
\hline $\begin{array}{l}\text { Los partidos y movimientos políticos motivan mi } \\
\text { participación }\end{array}$ & 45 & 47 & 8 \\
\hline No conocía las propuestas de los candidatos & 40 & 50 & 10 \\
\hline Ninguna propuesta me convenció & 44 & 45 & 11 \\
\hline Mi opinión es importante & 68 & 23 & 9 \\
\hline Las elecciones no me aportan nada & 39 & 51 & 10 \\
\hline Estoy desilusionado de la política & 55 & 35 & 10 \\
\hline Participar de las elecciones es un deber & 59 & 32 & 9 \\
\hline No me interesa la política & 48 & 43 & 10 \\
\hline Los políticos no me representan & 52 & 38 & 9 \\
\hline Todos los políticos son iguales & 51 & 40 & 9 \\
\hline El voto no es obligatorio & 54 & 37 & 13 \\
\hline $\begin{array}{l}\text { Tengo edad para votar pero no he sacado la cé- } \\
\text { dula }\end{array}$ & 24 & 63 & 12 \\
\hline No me interesa votar & 34 & 54 & \\
\hline
\end{tabular}

Tabla 6. Opiniones de los jóvenes en relación con las elecciones (\%)

Se destaca la alta apreciación de los jóvenes respecto a que participar en las elecciones es un derecho y un deber y la conciencia de que su opinión es importante. Un poco más de la mitad de los jóvenes considera que los políticos no los representan. De manera similar, 55\% dicen estar desilusionados de la política, aunque porcentualmente un poco menos $47 \%$ expresan que no les interesa la política. Sin embargo, más de la mitad considera que sí le interesa votar. La disparidad de estos resultados indica rechazo y distanciamiento de la política, de sus prácticas tradicionales y de sus actores, y al mismo tiempo muestran un alto grado de compromiso con el ejercicio ciudadano.

Los jóvenes manifiestan un mayoritario desacuerdo con la afirmación de que las elecciones no aportan nada, lo que las ubica como eventos importantes al parecer no sólo para ellos sino para la sociedad, catalogándolo como un gran deber de los ciudadanos, aunque vale acotar que en Colombia no es obligatorio.

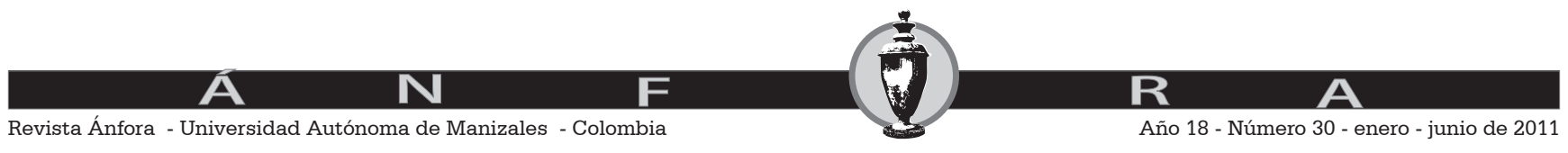


Los jóvenes de mayor edad consideran que los políticos no los representan, caso similar a la afirmación de que participar en las elecciones es un deber, opinión expresada por $55 \%, 58 \%$ y $64 \%$. Otro tanto ocurre con el planteamiento respecto a la desilusión con la política, progresión que va desde 53\%, 56\% a 57\%.

Cuando se afirma que los partidos y movimientos políticos motivan la participación, los jóvenes del Alto Oriente y Magdalena Caldense son los más críticos $5 \%$ y $12 \%$, mientras que en las demás subregiones se ubican con niveles de aceptación cercanos a la mitad de los consultados.

Si bien los jóvenes en las diferentes subregiones consideran en su mayoría que su opinión es importante entre $64 \%$ y $77 \%$, queda la inquietud de si se refieren a la manera como son valorados o a lo que desean que se les valore.

Cuando se afirma que las elecciones no aportan nada, siempre es mayoritario su desacuerdo, lo cual deja entrever que no es la elección como mecanismo lo que critican, sino el debilitamiento de la política y la pérdida de legitimidad de sus prácticas.

Las expresiones de los jóvenes de Caldas en relación con las elecciones, según las actividades que realizan y la ubicación geográfica, permiten afirmar que los que no estudian ni trabajan son mayoría sobre la afirmación "ninguna propuesta me convenció", "no me interesa la política", "todos los políticos son iguales", "los políticos no me representan", "estoy desilusionado de la política" y "no me interesa votar". Estas frases muestran que los jóvenes se han desencantado de la política.

\section{Conocimientos sobre algunas normativas y escenarios relacionados con los asuntos juveniles}

\begin{tabular}{|l|l|l|l|}
\hline Normativas e instituciones & $\mathrm{Si}$ & No & No responden \\
\hline $\begin{array}{l}\text { Conocimiento de la existencia de la Política Públi- } \\
\text { ca de Juventud en el Departamento de Caldas }\end{array}$ & 15 & 81 & 4 \\
\hline Conocimiento de la Ley General de la Juventud & 10 & 86 & 4 \\
\hline $\begin{array}{l}\text { Conocimiento acerca de los Consejos Municipales } \\
\text { de Juventud }\end{array}$ & 19 & 77 & 4 \\
\hline $\begin{array}{l}\text { Conocimiento acerca del funcionamiento del Con- } \\
\text { sejo de Juventud del Municipio }\end{array}$ & 24 & 59 & 17 \\
\hline $\begin{array}{l}\text { Conocimiento de la existencia del Observatorio } \\
\text { de Juventud }\end{array}$ & 7 & 87 & 6 \\
\hline
\end{tabular}

Tabla 7. Conocimiento de los jóvenes sobre la existencia de aspectos normativos e institucionales relacionados con ellos (\%).

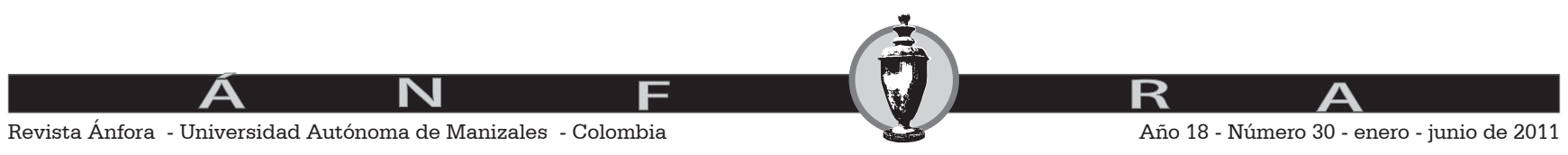


Los seres humanos no nacemos ciudadanos. La ciudadanía y la democracia son creaciones de las sociedades, por ello las sociedades democráticas ejercen un influjo educativo para que los ciudadanos ejerzan su ciudadanía. Para este ejercicio, estas sociedades garantizan unos estándares para que este ejercicio sea real. Entre estos estándares es prioritaria la garantía de haber resuelto las necesidades básicas de subsistencia, las posibilidades de movilización, el uso de las libertades a que se tiene derecho como ciudadano y el acceso a la información que se requiere para orientar las decisiones.

En este último aspecto, se ubica uno de las aspectos que debe acompañar a los jóvenes para que puedan participar en condiciones de equidad, puesto que el acceso y la utilización adecuada de la información, además de que sea una oportunidad importante para el ejercicio de la ciudadanía, cada vez más se convierte en una condición para que ello pueda ser así. El desconocimiento de las circunstancias en las cuales nos desenvolvemos cotidianamente impide un desempeño adecuado en la vida de la sociedad.

Aquí se ubica el interés por identificar el conocimiento que tienen los jóvenes sobre aspectos normativos e institucionales, marcan su quehacer en sociedad.

La gran mayoría de jóvenes desconoce la existencia de la Política Pública en el Departamento, sólo el 15,1\% de los jóvenes dicen conocerla, lo cual urge la necesidad de hacer realidad lo planteado en las conclusiones expresadas en el documento de Política Pública del Departamento de Caldas (2006), cuando al referirse a las conclusiones del área de la política se indica:

"Exaltan la falta de participación juvenil apoyada en la apatía y desconocimiento de la política, la falta de oportunidades de participación enigualdad decondiciones para todos los jóvenes de manera que no se privilegien los grupos particulares. Así mismo destacan la falta de oportunidades de participación organizadas que permitan a los jóvenes proponer, opinar y hacer veeduría de los asuntos públicos. Para los jóvenes esta es el área que requiere evaluación y replanteamiento de una oferta programática más articulada.

Las Alcaldías han apoyado con diferentes acciones los aspectos de esta área. Sin embargo, se han encontrado con un gran obstáculo que no tienen claro cómo superar, que es la apatía juvenil, la falta de interés manifestada por los jóvenes frente a las posibilidades de capacitación y participación que se les ofrece" (Política Pública de Juventud de Caldas, 2006):58. 
En relación con el conocimiento que tienen los jóvenes del Departamento de Caldas sobre la existencia de la Ley General de Juventud, sólo 10\% dice conocerla, de ahí que les ha llegado un poco más la política pública de Juventud que es departamental y más reciente, que la Ley nacional con más tiempo de vigencia. Lo anterior lleva a pensar que a pesar de las pretensiones de la Ley de la Juventud (en 1997), los jóvenes desconocen su, lo cual implica que hay mucho por construir en relación con los fines establecidos en ella, específicamente con respecto de la promoción de la formación integral de los jóvenes y el ejercicio pleno y solidario de la ciudadanía.

Los Consejos Municipales de Juventud salen mejor librados: el 19\% de los jóvenes sabe de la existencia de estos organismos que los representan. Sin embargo, la mayor parte no sabe cómo funcionan, como se observa en la tabla anterior. A su vez, los jóvenes que conocen el funcionamiento de estos organismos destacan los eventos que han organizado, los programas de atención y de prevención a las problemáticas juveniles, la presencia de algunos líderes y el respaldo que éstos les dan en algunas ocasiones. Por otro lado, dicen que no ven quiénes hacen parte de estas organizaciones, que no se dan a conocer y que no se enteran de las actividades que realizan.

El Observatorio de Juventud del Departamento de Caldas es poco conocido por los jóvenes. Sólo lo reconocen 7\%. Ello indica que esta es una entidad requiere mayor difusión entre los jóvenes, pues sus acciones no han llegado a ellos, tal como es lo deseable.

Por género y edad es equilibrado el desconocimiento que tienen los jóvenes sobre estos tópicos. Es notable que en Occidente Bajo sea mayor el conocimiento de la existencia de los Consejos Municipales de Juventud y saben más de su funcionamiento. Estos puntajes van del $26 \%$ y al $40 \%$, superando ostensiblemente a las demás subregiones.

Con respecto a la ubicación geográfica, el sector rural está más desinformado acerca de las normativas e instituciones que tienen que ver con los jóvenes, evidenciando diferencias de cerca del $5 \%$, con respecto de los jóvenes de la zona urbana.

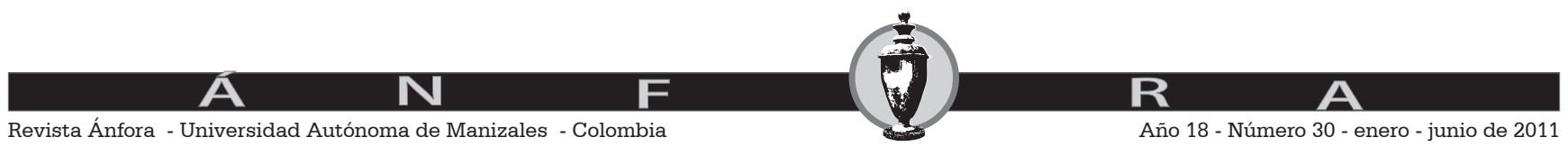




\section{Conclusiones}

Los resultados más relevantes de este estudio en relación con la participación política de los jóvenes, son los siguientes:

- Es muy bajo el interés de los jóvenes por la política tradicional, dado que la mayor parte dice interesarse poco o nada por estos asuntos.

- Los jóvenes expresan que se encuentran en un país democrático y, sin embargo, cuestionan las diferencias sociales, la corrupción, las formas de elegir a los gobernantes y el autoritarismo del gobierno.

- Los políticos y los partidos que ellos representan se han despreocupado de los asuntos de los jóvenes.

- La sociedad no ofrece espacios de participación y los jóvenes se apoyan en los partidos y movimientos políticos para acceder a los cargos públicos.

- No les interesan los partidos políticos y, sin embargo, conocen más a los oficialistas y menos a los de oposición.

- Valoran de manera importante las elecciones, las reconocen como mecanismo de acción política, aunque critican el debilitamiento de la política y la pérdida de legitimidad de sus prácticas.

- Paralos jóvenes, la política tradicional no es de su interés, está desacreditada, ha perdido legitimidad, es distante de sus intereses y de su vida cotidiana. No obstante, se sienten comprometidos con el ejercicio ciudadano.

- En su mayoría, los jóvenes desconocen las normativas que regulan sus posibilidades de acción social en asuntos como la Política Pública de Juventud del Departamento de Caldas, la Ley General de la Juventud y los Consejos de Juventud y el Observatorio de Juventud de Caldas.

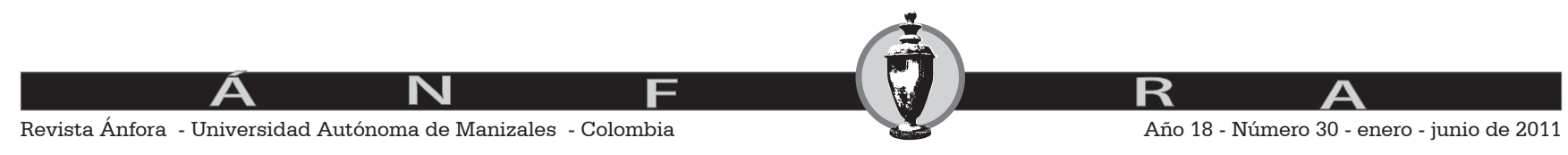




\section{Referencias}

CEPAL, OIJ, Naciones Unidas, AECID (2008). Juventud y cohesión social en Iberoamérica: un modelo para armar. Chile: Naciones Unidas.

CEPAL, OIJ (2007). La juventud Iberoamericana: Tendencias y urgencias. Buenos Aires: agosto, Segunda edición.

CEPAL, ECLAC. (2003). Capítulo I: Elementos para un Marco Conceptual y Contextual. En: Juventud, Población y Desarrollo en América Latina.

Congreso de Colombia (1997). Ley 375 de Julio 4 de 1997. Ley de la Juventud.

Constitución Política de Colombia 1991. URL (Consultado octubre, 2009): http://web.presidencia.gov.co/constitucion/index.pdf

DANE (2007). Encuesta sobre cultura política. Santa Fe de Bogotá.

De Souza Santos, Boaventura (2006). Para una democracia de alta intensidad, Capítulo III del texto Reinventar la emancipación social. Buenos Aires: Clacso,

Ferrater Mora, José (2004). Diccionario de filosofía. Barcelona: Ariel,

Gobernación del Departamento de Caldas (2006). Política Pública de Juventud del Departamento de Caldas. Manizales.

Gobernación de Caldas (2007). Política Pública de Juventud Decreto No. 0654 de 2007.

Mouffe, Chantal (2007). En torno a lo político. Buenos Aires: Fondo de Cultura Económica.

Zemmelman, Hugo. (2001). De la Historia a la Política. La Experiencia de América Latina. México: Editorial Siglo XXI. $3^{\text {a }}$ Edición 
